\title{
ENHANCEMENT OF THE IMMEDIATE RELEASE OF PARACETAMOL FROM ALGINATE BEADS
}

\author{
SAMAH HAMEDa, FARAH AMALINA AYOBa, MULHAM ALFATAMA ${ }^{b}$, ABD ALMONEM DOOLAANEA ${ }^{\mathrm{a}^{*}}$
}

aAdvanced Drug Delivery Lab, Department of Pharmaceutical Technology, Kulliyyah of Pharmacy, International Islamic University Malaysia, 25200 Kuantan, Pahang, Malaysia, bNon-Destructive Biomedical and Pharmaceutical Research Center, Faculty of Pharmacy, University Technology Mara, UiTM

Email: abdalmonemdoolaanea@yahoo.com

Received: 14 Oct 2016, Revised and Accepted: 02 Mar 2017

\section{ABSTRACT}

Objective: Alginate beads are not soluble in the acid leading to small portion released in the stomach. This may not be favourable for drugs administered for fast action like paracetamol. So, this study was aimed to increase the immediate release of paracetamol from alginate beads, i.e. the release in the acidic $\mathrm{pH}$.

Methods: The beads were prepared by dropwise paracetamol/alginate suspension in divalent cation solution. Two attempts were used to increase the dissolution of paracetamol in the acidic $\mathrm{pH}$. First attempt was by only changing preparation variables: needle size, alginate viscosity and drug loading using $2^{3}$ full factorial design. The second approach was by adding excipients like carbopol, tween and polyethylene glycol. The beads were characterized for their size, encapsulation efficiency and release profile.

Results: First approach, changing preparation variables without excipient adding, helped to increase the drug release in the acid but to a maximum of $26 \%$ using a smaller needle, lower drug loading and higher alginate viscosity. However, optimising the formulation with suitable excipients increased the drug release in the acid to $77.3 \%$. The optimised formulation included carbopol 940 (pH sensitive polymer) and tween 80 (facilitates water entry) in the beads, with using barium chloride instead of calcium chloride together with PEG 400 in the complexing solution.

Conclusion: To achieve immediate release of paracetamol from alginate beads in the acidic $\mathrm{pH}$, excipients need to be added. Rational selection of excipients is critical to achieve the desired drug release.

Keywords: Alginate beads, Paracetamol, Immediate release, Factorial design, Excipients

(c) 2016 The Authors. Published by Innovare Academic Sciences Pvt Ltd. This is an open access article under the CC BY license (http://creativecommons.org/licenses/by/4.0/)

DOI: http://dx.doi.org/10.22159/ijap.2017v9i2.15672

\section{INTRODUCTION}

The field of natural polysaccharides of marine origin is already large and expanding for the biomedical purpose. Alginate is one of the marine polysaccharides extracted from seaweeds and it is quite abundant in marine brown algae (Phaeophyceae). Other source of alginate is bacteria, where it is produced by only two types of bacterial genera: Pseudomonas and Azotobacter [1]. The alginic acid obtained can then be converted into a salt such as sodium alginate which is the major form currently used [2]. The favorable properties of alginates like biocompatibility, non-toxicity and ease of gelation led to their wide use in food and pharmaceutical industries as tablet disintegrant, thickening, and suspending agent, but the interest increased toward alginate gel beads as controlled-release devices for conventional drugs, proteins, microbial and biological cells [3].

Alginates are heteropolymer carboxylic acids linked by 1,4glycosidic bonds of p-D-mannuronic (M) and a-L-guluronic acid (G) units [4]. The most important feature of alginate's physical properties is the selective binding of multivalent cations that is the basis for gel formation. The gelation occurs by cross-linking of the uronic acid units with divalent cations to form the so-called 'eggbox' structure. Alginate's affinity is different toward the various divalent ions [5]. Calcium is the most used cation in the preparation of alginate beads [4]. Alginates isolated from different sources can vary both in monomer composition and block arrangement and these variations are also reflected in the properties of the alginate. Alginates with a high content of guluronic acid blocks give gels of considerably higher strength compared to alginates rich in mannuronate, as the $G$ residues exhibit a stronger affinity for divalent ions than the $\mathrm{M}$ residues. Transmittancy, swelling, and viscoelasticity of alginate gel membranes are highly affected by the $M / G$ ratio. The stiffness of alginate solution increases when the chain blocks increased in the order $\mathrm{MG}<\mathrm{MM}<\mathrm{GG}$ and when the molecular weight increases with increasing chain extension [6].

The nature of the crosslinking ion and cross-linking conditions such as gelation time and concentration of the divalent cation will affect the permeability of alginate gels and release the drugs from the beads [7].
Another factor also having an impact on alginate beads behaviour is the $\mathrm{pH}$ of the environment. The beads will stay for nearly $1-4 \mathrm{~h}$ in the stomach prior to their entry to the small intestine and then to the colon. The stay of beads in the highly acidic environment of the stomach may result in the hydrolysis of alginate into more soluble low molecular weight alginic acid. This causes a reduction in their mechanical strength and degree of crosslinking hence the beads may degrade at a faster rate after arriving at the colon[8]. In general, the properties of gel-forming depend on the monomeric composition, block structure, molecular size, concentration of polymer and the type of divalent metal ion [9].

Drug release from alginate beads depends on the swelling of the beads and the diffusion of the drug in the gel matrix. However, the alginate beads do not swell appreciably in acidic fluid while swell and disintegrate rapidly in the intestinal fluid leading to be convenient as delayed release formulation but not as immediate release one. Other materials (excipients) may need to be added to modulate the 'original' delayed release from the beads if the immediate release is required. A large number of factors including the chemical-physical properties of the raw materials (both drug and excipients) and the relative amounts of the components in the formulation can influence the drug release behavior from the final products [10].

Paracetamol has antipyretic, analgesic and weak anti-inflammatory actions[11]. It is sparingly soluble in water and prone to dissolution and bioavailability problems. Suspension dosage form of paracetamol may enhance this problem if properly formulated [12]. Paracetamol is not a classic biowaiver because it is classified as a BCS class III but it possesses properties borderline to Class I because the permeability is slightly below the cut-off value of $90 \%$, i.e. one study found permeability to be $80 \%$ once absorbed. This enables it to fulfil the requirements of a biowaiver [13] so the dissolution profile can be used to evaluate the in-vitro drug release profile. Paracetamol has a bitter taste and this taste is difficult to be masked in the liquid formulations like syrup and suspension. Encapsulation technologies are among several techniques to mask the bitter taste of drugs [14]. This study was conducted to encapsulate paracetamol in alginate beads so that 
later the beads can be used for taste masking. However, taste masking should not interfere with the drug release. High amount of immediate or burst release is required in many cases like pain relief or for some antibiotics like gentamicin formulated in microparticles [15]. Because alginate beads naturally produce delayed release beads, this study aimed to encapsulate paracetamol in alginate beads and enhance the drug release in the acidic environment so that the drug can give faster onset of action. At first, to increase the drug release in the acid, a method without adding excipients was attempted then another method was also attempted by adding some excipients. This study helps in understanding the effect of the formulation parameters and composition on the release of paracetamol in the acidic environment.

\section{MATERIALS AND METHODS}

\section{Chemicals and reagents}

Pharmaceutical grade paracetamol was obtained from Zhengjiang Kangle Pharmaceutical Co., Ltd. (Wenzhou, China). Sodium alginates of two viscosity grades noted here as high viscosity (896.44 mPa. s) and low viscosity (264.87 $\mathrm{mPa}$. s) were kindly offered from FMC Biopolymers (Philadelphia, USA). Calcium chloride and Tween 80 were obtained from Merck KgaA (Darmstadt, Germany). Other materials were of analytical grade.

\section{Methods}

\section{Preparation of paracetamol alginate beads}

Paracetamol was mixed with sodium alginate solution with the aid of sonicator probe. The beads were prepared simply by dropwise paracetamol/alginate suspension in divalent cation solution using syringe needle with stirring; the stirring was continued for approximately $5 \mathrm{~min}$ till the bead completely gelled then the beads were harvested by filtration, washed with distilled water and airdried for $15 \mathrm{~min}$.

\section{Optimisation of the beads without excipient addition}

The first approach to increase the release of paracetamol from alginate beads was changing some processing variables or the type of the ingredients without adding any new excipient. This is preferred, if successful because it reduces the number of excipients in the formulation and hence reducing the cost and complexity. Consequently, a total of 8 experiments was conducted to test 3 different parameters, namely: needle size, alginate viscosity grade and paracetamol loading as shown in table 1 . The experimental design followed $2^{3}$ full factorial design.

\section{Optimisation of the beads with excipient addition}

In another approach, the release of paracetamol from alginate beads was attempted by the addition of different excipients. These excipients change the complex of alginate (barium instead of calcium ions), change the viscosity in the acid (carbopol 940) or help in the water penetration into the beads such as Tween 80 and polyethylene glycol 400 (PEG 400). Four different formulations were prepared as presented in table 2 .

\section{Characterization of the beads}

The aim of this study is to optimize the release of paracetamol thus only basic characterization was performed on the beads. The bead size was measured using Vernier caliper on 10 beads. The encapsulation efficacy was estimated by quantifying the unencapsulated paracetamol in the calcium solution post-fabrication using spectrophotometer at $243 \mathrm{~nm}$.

\section{Release profile of paracetamol}

An accurately weighed amount of alginate beads equivalent to $34.7 \mathrm{mg}$ of paracetamol was added into $125 \mathrm{ml}$ of $\mathrm{HCl}$ buffer $\mathrm{pH} 1.2$ in conical flasks and introduced into incubator shaker (Innova 4000; New Brunswick Scientific Inc., Edison, NJ) with stirring at $100 \mathrm{rpm}$ and temperature at 37 ${ }^{\circ} \mathrm{C}$ for two h. At scheduled intervals $(15 \mathrm{~min}, 20 \mathrm{~min}, 30 \mathrm{~min}, 45 \mathrm{~min}, 1 \mathrm{~h}$ and $2 \mathrm{~h}$ ), 3-ml samples were taken and an equivalent volume of the fresh dissolution medium was added to keep the volume of release medium in the flask constant at $125 \mathrm{ml}$ and the amount of drug released was determined spectrophotometrically at $243 \mathrm{~nm}$ using Hitachi spectrophotometer model U-1900 (Hitachi, Tokyo, Japan). After $2 \mathrm{~h}$ time, the beads were transferred into $125 \mathrm{ml}$ phosphate buffer $\mathrm{pH} 6.8$ and the release profile was evaluated similarly.

\section{RESULTS AND DISCUSSION}

Paracetamol is usually given to the pediatric to lower the temperature and/or relief the pain. Therefore, it is aimed to give the action rapidly. One inherited issue with sodium alginate beads is that they tend to shrink in the acid environment and do not release the encapsulated material. The reason behind that is that sodium alginate is insoluble in acidic solution while soluble in alkaline solutions so when the calcium alginate beads is in an acidic environment ( $\mathrm{HCl}$ buffer), paracetamol releases slowly. The protonation of the carboxylate groups in an acidic environment reduced the electrostatic repulsions between alginate chains and led to shrinking of the beads and reduce paracetamol release [4]. So, this study is trying to increase the release percentage of paracetamol from the alginate beads in the acid environment of the stomach. To reduce the cost and complexity of the beads, the first approach was changing some processing variables or the type of the ingredients but not introducing any new material. The variables were testing using factorial design which is common experimental design in formulation optimization $[16,17]$. The size for the beads was dependent only on the needle size. Needle of $18 \mathrm{G}$ produced beads of size $1.81 \pm 0.05 \mathrm{~mm}$ whereas the $22 \mathrm{G}$ needle produced beads of size $1.28 \pm 0.06 \mathrm{~mm}$. Both are larger than the external diameter of the needle (18G: $1.27 \mathrm{~mm}, 22 \mathrm{G}: 0.71 \mathrm{~mm}$ ). This is attributed to the surface tension of the paracetamol-alginate suspension. Where there is no other mean to reduce the surface tension or pull the drop, the drop will build on the needle orifice until the weight become equal to the surface tension then it falls.

Table 1: Experimental design for optimisation the release of paracetamol without adding excipients, and the release properties of paracetamol-alginate beads after $2 \mathrm{~h}$ in $\mathrm{HCl}$ buffer. The numbers in bracket denote the coding of the factors in the factorial design

\begin{tabular}{|c|c|c|c|c|c|c|c|c|}
\hline $\begin{array}{l}\text { Experiment No./ } \\
\text { Parameters }\end{array}$ & Exp-1 & Exp-2 & Exp-3 & Exp-4 & Exp-5 & Exp-6 & Exp-7 & Exp-8 \\
\hline Needle size (gauge) & & $22 \mathrm{G}(2)$ & $22 \mathrm{G}(2)$ & $22 \mathrm{G}(2)$ & $22 \mathrm{G}(2)$ & 18G (1) & 18G (1) & 18G (1) \\
\hline Viscosity of alginate & High (2) & High (2) & Low (1) & Low (1) & High (2) & High (2) & Low (1) & Low (1) \\
\hline Drug loading (g paracetamol/15 ml alginate solution) & $5(2)$ & $5(2)$ & $1(1)$ & $5(2)$ & $1(1)$ & $1(1)$ & $1(1)$ & $5(2)$ \\
\hline $2 \mathrm{~h}$ cumulative release $(\%) \mathrm{HCl}$ buffer & $19.3 \pm 3.0$ & $23.1 \pm 3.3$ & $11.7 \pm 0.6$ & $17.7 \pm 1.8$ & $18.5 \pm 1.5$ & $26.0 \pm 4.3$ & $7.2 \pm 1.3$ & $14.0 \pm 4.7$ \\
\hline
\end{tabular}

The release percentage after $2 \mathrm{~h}$ in $\mathrm{HCl}$ buffer is shown in table 1. Factorial analysis as shown in fig. 1 revealed that the effect of alginate viscosity and drug loading on drug release in acid were significant $(\mathrm{p}<0.05)$. All 2 -way and 3 -way interactions were also significant $(p<0.05)$. As shown in fig. 1 , alginate viscosity has the highest effect on drug release in the acid. The alginate of higher viscosity released paracetamol faster than lower viscosity alginate, which is an unexpected result. It was expected that higher viscosity alginate produces stronger beads and so the release becomes slower. However, the higher viscosity alginate may produce non- homogenous beads due to a strong shell made at the beginning of complexation; leading to less calcium in the core. On the other hand, lower viscosity alginate allows calcium ions to enter to the core, making homogenous beads. This might explain the faster release observed by higher viscosity alginate. Higher drug loading resulted in higher release (fig. 1).

Even though the interactions were statistically significant $(\mathrm{p}<0.05)$ but only the interaction between alginate viscosity and drug loading was of important change on the drug release (fig. 1B right panel). 
When alginate viscosity increased, drug release increased in both drug loading values, but the increase was less with the higher drug loading (5\%). Other interactions showed a slight increase or decrease on the drug release as shown in fig. 1B.

A

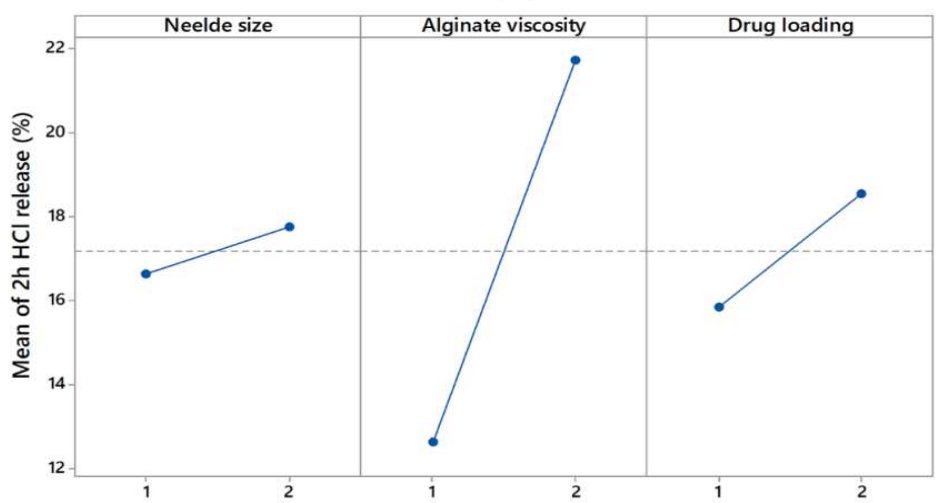

B

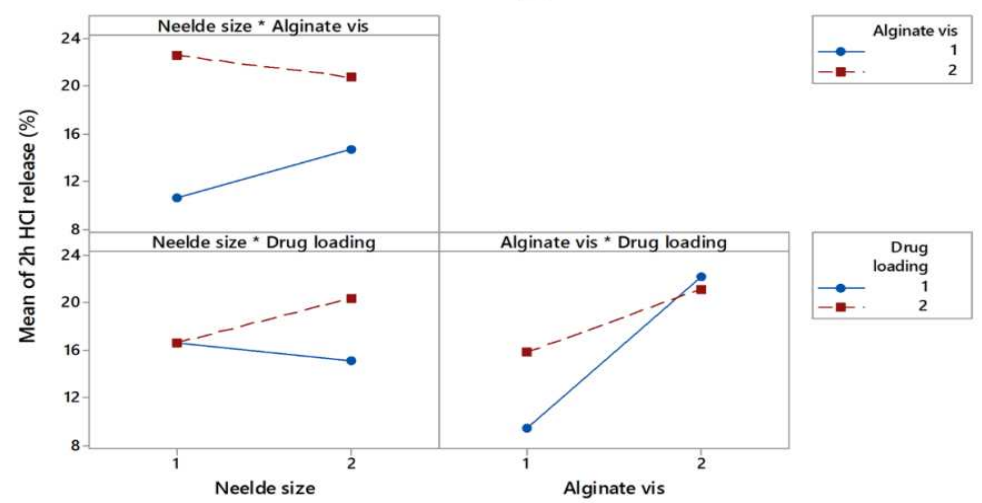

Fig. 1: Effect of needle size, alginate viscosity and drug loading on the release percentage of paracetamol after $2 \mathrm{~h}$ in the acid. A: main effect, B: interactions. vis: viscosity

The release profile in both $\mathrm{HCl}$ buffer and phosphate buffer is presented in fig. 2. The release in the acidic environment ranged from $7.2 \pm 1.3 \%$ (Exp-7) to $26.0 \pm 4.3 \%$ (Exp-6) while the release in the phosphate buffer occurred rapidly and all formulations released the total amount of paracetamol within 2 h. Actually, all formulations, except for Exp-4 and Exp-8, released almost $80 \%$ or more within 15 min. The two formulations, Exp-4 and Exp-8, released around 57\% within 15 min then gradually released the remaining amount within $2 \mathrm{~h}$.

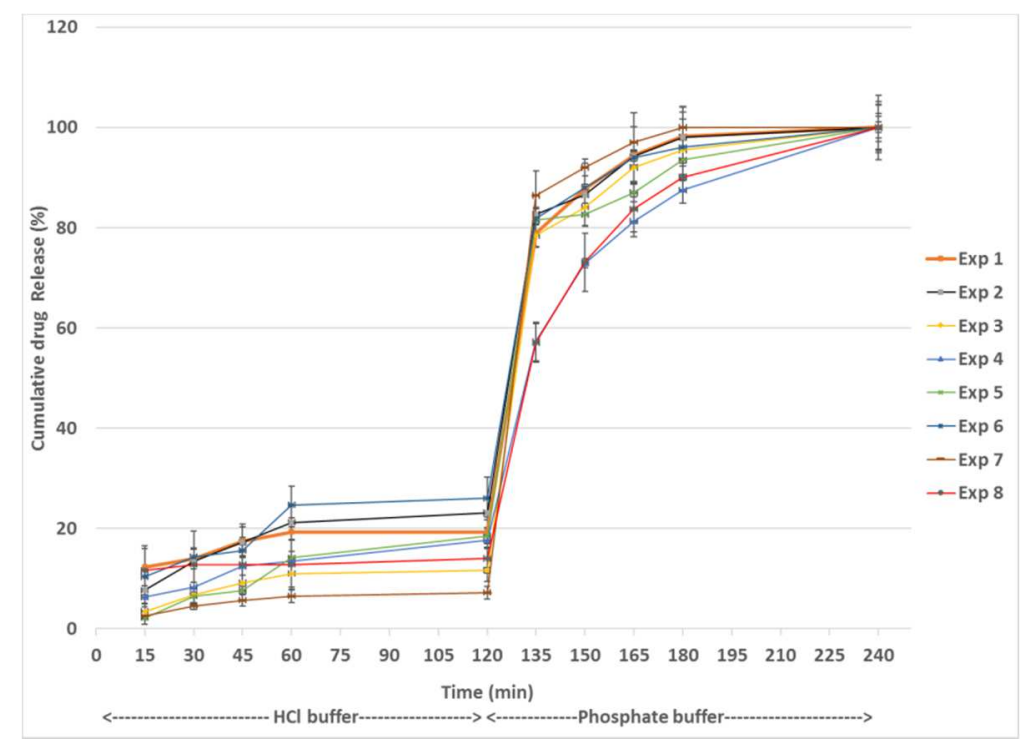

Fig. 2: Release profile of paracetamol from alginate beads in both $\mathrm{HCl}$ and phosphate buffers. Alginate formulations were prepared without excipient addition. Refer to table 1 for the parameters in each formulation. Each point represent mean (3 samples) \pm standard deviation (SD) 
To sum up, the optimisation without excipient addition helped to increase the release of paracetamol in the acidic buffer but only to $26.0 \%$ of the total encapsulated amount. This may not be sufficient to give the desired fast onset of action. Therefore, another approach was attempted to increase the release of paracetamol in the acidic environment. This approach involves the addition of different excipients as shown in table 2.

Table 2: Experimental design for optimization the release of paracetamol with adding excipients. The ingredients were used in the following concentrations/quantities: paracetamol (1g), carbopol 940 (1\%), tween 80 (1\%), calcium chloride (1\%), barium chloride (1\%), PEG 400 (25\%). The preparation was carried out using $22 \mathrm{G}$ needle

\begin{tabular}{|c|c|c|c|c|}
\hline Medium & Formulation 1 & Formulation 2 & Formulation 3 & Formulation 4 \\
\hline \multirow[t]{4}{*}{ Paracetamol/alginate suspension } & -Paracetamol & -Paracetamol & -Paracetamol & -Paracetamol \\
\hline & -Sodium alginate & -Sodium alginate & -Sodium alginate & -Sodium alginate \\
\hline & & -Carbopol 940 & -Carbopol 940 & -Carbopol 940 \\
\hline & & -Tween 80 & -Tween 80 & -Tween 80 \\
\hline Divalent cation solution & -Calcium chloride & -Barium chloride & $\begin{array}{l}\text {-Barium chloride } \\
\text {-PEG } 400\end{array}$ & $\begin{array}{l}\text {-Calcium chloride } \\
\text {-PEG } 400\end{array}$ \\
\hline 2 h cumulative release (\%) $\mathrm{HCl}$ buffer & 10.5 & 26.5 & 77.3 & 20.4 \\
\hline
\end{tabular}

The release profiles of paracetamol from the four formulations were different as illustrated in fig. 3. The maximum release of paracetamol was observed in Formulation 3, which reached about 41\% after 15 min and increased gradually to $63.7 \%$ after one hour and $77.3 \%$ after two hours. Meanwhile, the other formulations did not increase the release of paracetamol better than the optimisation without excipient addition.

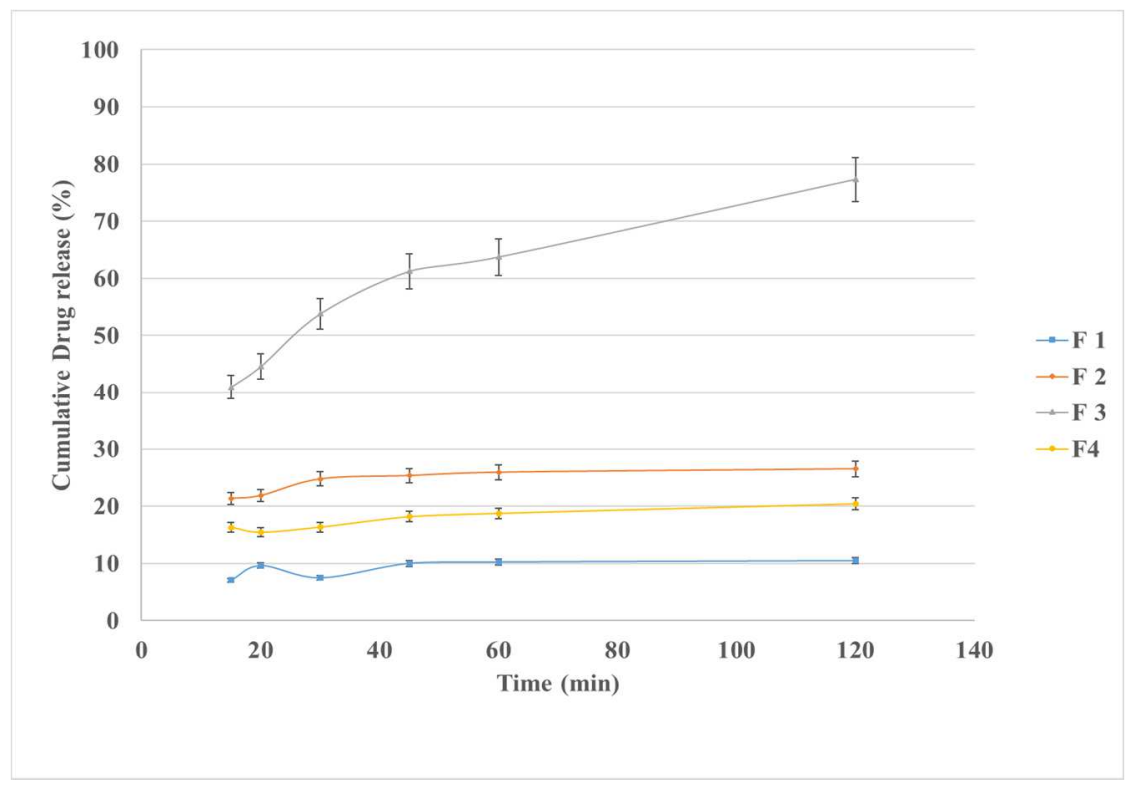

Fig. 3: Release profile of paracetamol from alginate beads in $\mathrm{HCl}$ buffer. Alginate formulations were prepared with excipient addition. Refer to table 2 for the composition of each formulation. Each point represent mean (3 samples) \pm standard deviation (SD)

In Formulation 2, compared to Formulation 1, the paracetamolalginate suspension was enhanced by the addition of Carbopol 940 and Tween 80 whereas in the divalent solution calcium ions were replaced by barium ions. Carbopol 940 is a synthetic crosslinked high molecular weight polymer of acrylic acid. It is hygroscopic, disperse in water and makes gels when neutralized to $\mathrm{pH} \sim 6.0$. However, the viscosity drops considerably in the pH less than 3 [18]. This property was the rationale behind the addition of Carbopol to the formulation. Carbopol is dissolved with sodium alginate then incorporated in the beads. Upon contact with the acidic $\mathrm{pH}$ of the stomach, the viscosity of carbopol decreases and hence the bead structure becomes weaker. Consequently, more entrapped material (paracetamol) can release to the medium, contributing to the more release observed. Tween 80 is a hydrophilic surfactant, commonly used as wetting agent or emulsifier. It may help to allow more water and acid to penetrate the beads. It also helps in wetting paracetamol particles and facilitates its solubility. Barium ions were shown previously to make the beads swell more [5], and this will facilitate the release of paracetamol. Previous studies showed that using barium ions as crosslinker resulted in the faster release of the encapsulated material $[19,20]$. All these excipients helped to increase the release of paracetamol in the acid by about 2.5 folds. Despite that the release was still less than $30 \%$ which is considered low.

The addition of PEG400 (Formulation 3) to the ionic solution resulted in dramatic increase in the release of paracetamol in the acid, from $26.5 \%$ to $77.3 \%$. PEG 400 is water miscible solvent in all proportions. During the complexation, PEG is expected to penetrate inside the beads and coat the surface. Then, upon contact with the acidic environment, PEG leaks into the medium disturbing the bead structure and allowing water to penetrate and paracetamol to release. The observed effects of PEG comply with previous studies that reported the presence of polyethene glycols in the film coats, especially the liquid grades like PEG 400, tended to increase the water penetration and reduce the protection of the enteric film coat against the acidic $\mathrm{pH}$ [18].

\section{CONCLUSION}

To achieve the immediate release of paracetamol from alginate beads in the acidic $\mathrm{pH}$, excipients need to be added. Calcium alginate beads are not soluble in the acidic stomach solution leading to a small percentage of paracetamol released. The optimisation of drug 
release by changing variables such as alginate viscosity, needle size and drug loading (no excipient is added) failed to increase the paracetamol release in the acid more than $26.0 \pm 4.3 \%$. Adding suitable excipients such as carbopol 940 and tween 80, and using barium chloride instead of calcium chloride together with a coating with PEG 400 helped to increase the drug release in the acid up to $77.3 \%$. This increase in drug release in the acidic $\mathrm{pH}$ may help to get the faster onset of the pharmacological action of paracetamol.

\section{ACKNOWLEDGMENT}

This project is supported by IIUM RESEARCH INITIATIVE GRANT (RIG) THEME (RIGS15-092-0092).

\section{CONFLICTS OF INTERESTS}

Declare none

\section{REFERENCES}

1. Ahmed N. Genetics of bacterial alginate: alginate genes distribution, organisation and biosynthesis in bacteria. Curr Genomics 2007;8:191-202.

2. Smidsrod 0, Draget K. Chemistry and physical properties of alginates; 1996.

3. Aslani P, Kennedy R. Effect of gelation conditions and dissolution media on the release of paracetamol from alginate gel beads. J Microencapsul 1996;13:601-14.

4. Velings NM, Mestdagh MM. Physico-chemical properties of alginate gel beads. Polym Gels Networks 1995;3:311-30.

5. Mørch ÝA, Donati I, Strand BL, Skjåk-Bræk G. Effect of Ca2+, Ba2+, and Sr2+on alginate microbeads. Biomacromolecules 2006; 7:1471-80.

6. Draget KI, Smidsrød 0, Skjåk-Bræk G. Alginates from algae. Biopolymers Online; 2005.

7. Aslani P, Kennedy RA. Studies on diffusion in alginate gels. I. Effect of cross-linking with calcium or zinc ions on the diffusion of acetaminophen. J Controlled Release 1996;42:75-82.

8. Bajpai S, Sharma S. Investigation of swelling/degradation behaviour of alginate beads crosslinked with $\mathrm{Ca} 2+$ and $\mathrm{Ba}$ 2+ions. React Funct Polym 2004;59:129-40.

9. Martinsen A, Skjåk-Bræk G, Smidsrød O. Alginate as immobilisation material: I. Correlation between chemical and physical properties of alginate gel beads. Biotechnol Bioeng 1989;33:79-89.
10. López-Cacho J, González-R PL, Talero B, Rabasco A, GonzálezRodríguez M. Robust optimization of alginate-carbopol 940 bead formulations. Sci World J 2012. http://dx.doi.org/10.1100/2012/605610

11. Forrest JA, Clements J, Prescott L. Clinical pharmacokinetics of paracetamol. Clin Pharmacokinet 1982;7:93-107.

12. Azam MG, Haider SS. Evaluation of dissolution behaviour of paracetamol suspensions. Dhaka Univ J Pharm Sci 2008;7:53-8.

13. Kalantzi L, Reppas C, Dressman J, Amidon G, Junginger H, Midha $\mathrm{K}$, et al. Biowaiver monographs for immediate release solid oral dosage forms: acetaminophen (paracetamol). J Pharm Sci 2006;95:4-14.

14. Coupland J, Hayes J. Physical approaches to masking bitter taste: lessons from food and pharmaceuticals. Pharm Res 2014;31:2921-39.

15. Ismail H, Fahmi A, Doolaanea AM, Awang M, Mohamed F. High initial burst release of gentamicin formulated as PLGA microspheres implant for treating the orthopaedic infection. Int J Pharm Pharm Sci 2012;4:685-91.

16. Sakhare SS, Yadav AV, Jadhav PD. Design, development and characterization of mucoadhesive gastro spheres of carvedilol. Int J Appl Pharm 2016;8:37-42.

17. Butani S, Shah T, Parmar K, Rajput A. Development ofrizatriptan benzoate microspheres for the nose to brain targeting. Int J Appl Pharm 2016;8:69-74.

18. Rowe RC, Sheskey PJ, Weller PJ. Handbook of pharmaceutical excipients: Pharmaceutical Press: London; 2006.

19. Hariyadi DM, Hendradi E, Purwanti T, Fadil FDGP, Ramadani CN. Effect of crosslinking agent and polymer on the characteristics of ovalbumin-loaded alginate microspheres. Int J Pharm Pharm Sci 2014;6:469-74.

20. El-menshawe SF, Kharshoum RM, Hamad DS, Halawa A. Effect of biodegradable copolymers and divalent cations on the sustained release ability of propranolol hydrochloride loaded biomaterial microspheres; 2016. p. 7.

\section{How to cite this article}

- Samah Hamed, Farah Amalina Ayob, Mulham Alfatama, Abd Almonem Doolaanea. Enhancement of the immediate release of paracetamol from alginate beads. Int J Appl Pharm 2017;9(2):47-51. 